\title{
Deficiencias de nutrientes en suelos semiáridos. I: Ajuste de un método en invernáculo ${ }^{1}$
}

\begin{abstract}
1 Proyecto P-528905 financiado por la Secretaria de Ciencia y Técnica de la UNSL
2 Area de Recursos Naturales, Facultad de Ingeniería de la Universidad Nacional de San Luis (UNSL), 25 de mayo 384, (5730) V. Mercedes (SL), Argentina, E-mail: barbosa@fices.unsl.edu.ar (Foto)

${ }^{3}$ Area de Recursos Naturales, Facultad de Ingeniería UNSL
\end{abstract}

Protocolo $118-6 / 8 / 2001$

\begin{abstract}
Resumen: El presente estudio fue llevado a cabo con el objetivo de estimar las carencias de nutrientes minerales y sus prioridades en suelos agrícolas de regadío del área de influencia de Villa Mercedes (San Luis, Argentina), utilizando una técnica simple que combina métodos sustractivos y aditivos en invernáculo. Las experiencias se realizaron comparando ocho tratamientos sustractivos (C, C-Mg, C-Ca, C-P, C-K, C-S, C-EM, y T), y cuatro aditivos (N, NP, NS, y NPS), en un Haplustoll éntico a dos profundidades $(0-20$ y $20-40 \mathrm{~cm})$ empleando como planta índice Lolium multiflorum Lam. Los resultados muestran que: 1) no existen deficiencias nutritivas de $\mathrm{K}$, $\mathrm{Mg}$, Ca ni EM (elementos menores), en las profundidades estudiadas, 2) el S fue deficiente en las dos profundidades, 3) el $\mathrm{P}$ presenta una disminución de rendimientos en superficie mientras que es deficiente en profundidad. Asimismo se obtuvo una correlación positiva $(r=0,9128)$ entre el consumo de agua realizada por el cultivo y los rendimientos de materia seca obtenidos de los diferentes tratamientos.
\end{abstract}

Palabras clave: nutrientes, deficiencias, método sustractivo, método aditivo, suelos

\section{Nutrient deficiencies in semiarid soils. I: Adjustment of a method in greenhouse}

\begin{abstract}
This study was carried out in order to estimate the mineral nutrient deficiencies and their priorities in irrigated arable soils in the area of Villa Mercedes (San Luis, Argentine) by means of a simple technique, which combines subtractive and additive methods in greenhouse. The trials were performed by comparing eight subtractive treatments (C, C-Mg, C-Ca, C-P, C-K, C-S, C-ME and T) and four additive ones (N, NP, NS and NPS), in an Entic Haplustoll, at two depths $(0-20$ and $20-40 \mathrm{~cm})$ and using Lolium multiflorum Lam. as index plant. The achieved results show that: 1) there are no nutrient deficiencies of $\mathrm{K}, \mathrm{Mg}, \mathrm{Ca}$, or $\mathrm{ME}$ (minor element), in depths studied; 2) S was deficient in both depths; 3) P presents a marked decrease of yield at surface level while it is found to be deficient at greater depths. In addition, there appeared a positive correlation ( $r=0.9128$ ) between the water consumed by the crop and the yields resulting from the different treatments.
\end{abstract}

Key words: nutrients, deficiencies, subtractive method, additive method, soils

\section{INTRODUCCIÓN}

Existen en la actualidad tres tipos de métodos para el estudio de las deficiencias de nutrientes en los suelos: "sustractivos", por eliminación selectiva de los mismos (Chaminade, 1964; Priano et al., 1983; Echeverría \& Navarro, 1983; Pilatti et al., 1988), "aditivos", adiccionando los elementos nutritivos (Echeverría, 1985; Barbosa et al., 1997), y "analíticos" investigando el contenido de los elementos en las diferentes partes vegetales (Jones \& Case, 1990; Sims, 2000). Se seleccionaron los dos primeros por ser los que permiten trabajar con los suelos tal como se encuentran en la naturaleza.

Si bien estos métodos fueron bastante empleados en las décadas pasadas, aún cuando en la actualidad su uso se encuentra un tanto restringido, sus ventajas son indudables para estudios en invernáculos, ya que permiten trabajar con muestras de suelos naturales y aportar una información, que aún cuando en sí misma puede que no sea suficiente, siempre resultará complementaria de la aportada por otras metodologías. Por otro lado, dada la dificultad que entraña el estudio de cada 
uno de los factores, siempre será de utilidad el poder disponer de diferentes métodos que aporten información concurrente. Estas técnicas que, aunque introducen un cierto grado de manipulación sobre las condiciones naturales (difícilmente evitable en cualquier estudio bajo condiciones de invernáculo), se aproxima más que las otras a la situación que de hecho se dá naturalmente.

A partir del trabajo original de Chaminade (1964), se realizaron numerosos estudios a nivel mundial para la aplicación de esta técnica, sobresaliendo los de Schenkel et al. (1971). En Argentina, la metodología fue aplicada en suelos pampeanos por Romero et al. (1973), utilizando como planta índice alfalfa. Posteriormente, Echeverría \& Navarro (1983) la aplicaron en suelos de la provincia de Buenos Aires, Priano et al. (1983) y Pilatti et al. (1988) en suelos de la provincia de Santa Fé. Los antecedentes más cercanos a la área en estudio se encuentran en trabajos realizados para la zona Central de Córdoba por Fisher et al. en la década del 70.

El área de muestreo de este estudio comprende un sector irrigado de aproximadamente unas 10.000 ha sobre las terrazas del Río Quinto en los alrededores de la ciudad de Villa Mercedes (SL). En esta porción se ha modificado el equilibrio natural producto de la acción del hombre sobre el suelo para la obtención de diferentes cultivos (maíz, sorgo, girasol, hortalizas bajo riego, etc) o por la utilización del pastizal natural como fuente de alimentación para el ganado. Este aprovechamiento ha producido diferentes tipos de degradación, siendo la pérdida de fertilidad del suelo de vital importancia para la continuidad de las explotaciones agropecuarias de manera sustentable.

Sin embargo, trabajos sobre la fertilidad de estos suelos son escasos, mencionando el realizado por el INTA San Luis sobre Pasto Llorón (d'Hiriart \& Marchi, 1978) en un suelo vecino. Asimismo las Cartas de suelo aportan muy poco en relación a la fertilidad de dichos suelos (Barbosa et al., 1997).

El objetivo del presente trabajo fue estimar las deficiencias de nutrientes y su prioridad en los suelos utilizando el cultivo de 'rye grass' (Lolium multiflorum Lam.) como planta índice bajo condiciones de invernáculo.

\section{MATERIAL Y MÉTODOS}

Se utilizaron macetas que fueron llenadas con $1 \mathrm{~kg}$ de suelo seco al aire y tamizado (tamiz de $2 \mathrm{~mm}$ ) tomado entre una profundidad 0-20 y $20-40 \mathrm{~cm}$, de una terraza proxima al río Quinto. El suelo fue clasificado como Haplustoll éntico, mixto, grueso, térmico serie Lavaisse, cuyas características físicoquímicas se detallan en el Tabla 1.

Se emplearon macetas de plástico cilíndricas, de aproximadamente $1000 \mathrm{~cm}^{3}$ de capacidad, sin drenaje. Los riegos se efectuaron con agua destilada, procurando mantener, a través de pesadas, durante el periodo vegetativo, un porcentaje de agua entre humedad equivalente y sus dos tercios.

Se utilizó ray grass como planta índice, por la facilidad de manejo, velocidad de crecimiento y capacidad de rebrote, a razón de $4 \mathrm{~g}$ de semillas por maceta. De esta manera fue posible lograr de cuatro a seis cortes sucesivos con lo que se calculó el nivel de reservas de nutrientes en el suelo y su agotamiento en el transcurso del tiempo entre los cortes.
Tabla 1. Características físico-químicas del suelo utilizado

\begin{tabular}{|c|c|c|c|}
\hline \multirow{2}{*}{ Caracteristica } & & Ap & $\mathrm{AC}$ \\
\hline & & $0-22 \mathrm{~cm}$ & $22-60 \mathrm{~cm}$ \\
\hline \multirow{3}{*}{$\begin{array}{l}\text { Materia } \\
\text { Orgánica }\end{array}$} & Carbono $\left(\mathrm{g} \mathrm{kg}^{-1}\right)$ & 8,9 & 4,6 \\
\hline & Nitrógeno $\left(\mathrm{g} \mathrm{kg}^{-1}\right)$ & 1,0 & 0,5 \\
\hline & Relación $\mathrm{C} / \mathrm{N}$ & 8,90 & 9,20 \\
\hline \multirow{9}{*}{$\begin{array}{l}\text { Granulometría } \\
\qquad\left(\mathrm{g} \mathrm{kg}^{-1}\right)\end{array}$} & Arcilla $(<2 \mu \mathrm{m})$ & 102,8 & 82,6 \\
\hline & $\operatorname{Limo}(2-20 \mu \mathrm{m})$ & 52,4 & 78,0 \\
\hline & Limo $(20-50 \mu \mathrm{m})$ & 136,8 & 155,3 \\
\hline & Arena $(50-75 \mu \mathrm{m})$ & 236,0 & 189,4 \\
\hline & Arena $(75-100 \mu \mathrm{m})$ & 200,3 & 198,1 \\
\hline & Arena $(100-250 \mu \mathrm{m})$ & 308,0 & 357,2 \\
\hline & Arena $(250-500 \mu \mathrm{m})$ & 10,8 & 10,7 \\
\hline & Arena $(500-1000 \mu \mathrm{m})$ & 4,9 & 6,3 \\
\hline & Arena $(1-2 \mathrm{~mm})$ & 0,4 & 0,4 \\
\hline \multicolumn{2}{|c|}{ Textura } & \multicolumn{2}{|c|}{ Franco arenosa Franco arenosa } \\
\hline \multicolumn{2}{|c|}{ Humedad Equivalente $\left(\mathrm{g} \mathrm{kg}^{-1}\right)$} & 139,2 & 122,1 \\
\hline \multicolumn{2}{|c|}{ Calcáreo $\left(\mathrm{CaCO}_{3} \mathrm{~g} \mathrm{~kg}^{-1}\right)$} & 3,5 & 3,6 \\
\hline \multicolumn{2}{|c|}{$\mathrm{pH}$ en pasta } & 6,21 & 6,95 \\
\hline \multicolumn{2}{|c|}{$\mathrm{pH}$ en agua $1: 2,5$} & 6,99 & 7,28 \\
\hline \multicolumn{2}{|c|}{$\mathrm{pH}$ en $\mathrm{ClK} 1 \mathrm{~N} 1: 2,5$} & 5,97 & 6,28 \\
\hline \multicolumn{2}{|c|}{ Resistencia de la pasta $(\Omega)$} & 9296 & 11620 \\
\hline \multicolumn{2}{|c|}{ Valor $\mathrm{T}\left(\mathrm{Cmol}_{+} \mathrm{kg}^{-1}\right)$} & 8,20 & 7,30 \\
\hline \multicolumn{2}{|c|}{ Valor $\mathrm{H}\left(\mathrm{Cmol}+\mathrm{kg}^{-1}\right)$} & 1,40 & 0,80 \\
\hline
\end{tabular}

El estudio del suelo superficial $(0-20 \mathrm{~cm})$ incluyó ocho tratamientos sustractivos y cuatro aditivos, todos en diseño de bloques completamente aleatorizados, con tres repeticiones para todos ellos, en dos dosis diferentes (50 y 33\% de la concentración original). El período del cultivo abarcó 147 y 154 d, durante el cual se realizaron 5 y 6 cortes, respectivamente. En el suelo subsuperficial (20-40 cm de profundidad) se utilizó una de las concentraciones (33\%) con idénticos tratamientos y diseño, con un período de $114 \mathrm{~d}$, obteniéndose cuatro cortes.

Se realizó la rotación periódica de los recipientes dentro de cada repetición, a los fines de atenuar el efecto de bordura en todos los ensayos.

Debido al poco contenido de materia orgánica y a la baja capacidad de intercambio catiónico de los suelos considerados y a que el rendimiento disminuye rápidamente a cero en ausencia de un abono nitrogenado (observado en experiencias anteriores por Galarza et al., 1993), no se realizó el tratamiento completo menos nitrógeno (C-N). Las dosis se ajustaron debido a estas dos circunstancias anteriores. El $\mathrm{N}$ fue fraccionado y colocado semanalmente a razón de $20 \mathrm{~mL}$ de una solución de nitrato de amonio $\left(4,2875 \mathrm{~g} . \mathrm{L}^{-1}\right)$ antes del riego. Las fuentes nutritivas, las concentraciones empleadas y los tratamientos se presentan en las Tablas 2 y 3.

Se preparó un solo tratamiento para el conjunto de los elementos menores (EM). Previendo que, si las deficiencias nutritivas minerales hubiesen sido observadas, se podría recurrir a un ensayo separando los mismos. Dentro del ensayo, el Testigo (T) es un tratamiento de control, ya que únicamente se lo riega con agua destilada y no posee aportes de soluciones nutritivas.

La evaluación de la producción de materia seca (MS) se realizó mediante cortes periódicos, los que fueron secados en estufa de aire forzado; expresando los resultados en gramos de materia seca a $60^{\circ} \mathrm{C}$. 
Tabla 2. Dosis y fuentes empleadas

\begin{tabular}{clrr}
\hline \multirow{2}{*}{ Elemento } & \multirow{2}{*}{ Fuente Nutritiva Utilizada } & \multicolumn{2}{c}{ Dosis $(\mathrm{g})$} \\
\cline { 3 - 4 } & & $50 \%$ & $33 \%$ \\
\hline $\mathrm{P}$ & Fosfato monosódico & 48,575 & 32,383 \\
$\mathrm{~K}$ & Bicarbonato de Potasio & 53,175 & 35,450 \\
$\mathrm{Ca}$ & Nitrato de Calcio & 36,875 & 24,583 \\
$\mathrm{Mg}$ & Cloruro de Magnesio & 12,685 & 8,456 \\
$\mathrm{~S}$ & Sulfato de Sodio & 25,000 & 16,666 \\
$\mathrm{~N}$ & Nitrato de Amonio & 12,500 & 8,333 \\
$\mathrm{Mn} *$ & Sulfato de Manganeso & 0,450 & 0,300 \\
$\mathrm{Zn} *$ & Sulfato de Zinc & 0,222 & 0,148 \\
$\mathrm{Cu} *$ & Sulfato de Cobre & 0,315 & 0,208 \\
$\mathrm{Bo}^{*}$ & Acido Bórico & 0,200 & 0,133 \\
$\mathrm{Mo}^{*}$ & Molibdato de Amonio & 0,016 & 0,011 \\
\hline * Elementos menoresm & & \multicolumn{2}{c}{}
\end{tabular}

Tabla 3. Tratamientos utilizados

\begin{tabular}{ccl}
\hline \multirow{4}{*}{ Simbolos } & \multicolumn{1}{c}{ Tratamientos } \\
\cline { 2 - 3 } & $\mathrm{T}$ & \multicolumn{1}{c}{ Testigo } \\
\hline \multirow{4}{*}{ Sustrativos } & C & Completo \\
& C-P & Completo menos Fósforo \\
& C-Ca & Completo menos Potasio \\
& C-Mg & Completo menos Calcio \\
& C-S & Completo menos Magnesio \\
& C-EM & Completo menos Azufre \\
Aditivos & N & Nitrógeno menos Elementos Menores \\
& NS & Nitrógeno más Azufre \\
& NP & Nitrógeno más Fósforo \\
& NPS & Nitrógeno más Fósforo más Azufre \\
\hline
\end{tabular}

Con los resultados acumulados obtenidos de cada corte y el consumo de agua que realizó el cultivo, se efectuó un análisis de varianza, aplicando posteriormente el test de Tukey para comparación de medias una vez comprobada la homogeneidad de la varianza por medio del test de Bartlett's. También se realizaron diferentes correlaciones.

Se elaboraron índices de rendimiento del ensayo con el objeto de obtener otra interpretación analítica de los resultados.

\section{RESULTADOS Y DISCUSION}

No fueron observados síntomas de deficiencia de nutrientes ni de salinidad en los tratamientos. Por otro lado los resultados da MS acumulada al quinto corte del suelo superficial muestran que existe una alta correlación $\left(\mathrm{R}^{2}=0,994\right)$ entre las dos concentraciones utilizadas confirmando la eficiencia de las mismas tanto en los tratamientos sustrativos como aditivos. También se encontró una correlación positiva $\left(\mathrm{R}^{2}=0,9128\right)$ entre la producción de MS de ray grass y el consumo de agua realizado por el cultivo. Este hecho determina una ventaja comparativa ya que resulta más fácil esta medición por parte del productor. Asimismo, en la interpretación de los resultados es importante tener presente el hecho de que el agotamiento del suelo se produce más intensivamente en las macetas que en el campo (Barbosa et al., 1997).

En las Tablas siguientes se observan los promedios de materia seca de cada corte y se indican las diferencias significativas para las dos profundidas estudiadas.

No fueron observadas diferencias significativas de MS en las dos profundidades en los tratamientos C-K, C-Ca, C-Mg y
Tabla 4. Promedio de materia seca para cada corte, concentración al $33 \%$, suelo $0-20 \mathrm{~cm}$

\begin{tabular}{|c|c|c|c|c|c|}
\hline \multirow{2}{*}{ Tratamiento } & \multicolumn{5}{|c|}{ Promedios de los Cortes (g) } \\
\hline & Primero & Segundo & Tercero & Cuarto & Quinto \\
\hline \multicolumn{6}{|c|}{ A. Profunidade $0-20 \mathrm{~cm}$} \\
\hline $\mathrm{T}$ & $0,4552 * *$ & $0,3019 * *$ & $0,3314 * *$ & - & - \\
\hline $\mathrm{C}$ & 0,6930 & 1,0797 & 1,8339 & 2,2943 & 1,6582 \\
\hline $\mathrm{C}-\mathrm{P}$ & 0,7041 & $0,8043 * *$ & $1,5106 * *$ & $2,0594 * *$ & $1,5262 * *$ \\
\hline $\mathrm{C}-\mathrm{K}$ & 0,7901 & 1,0580 & 1,7226 & 2,0276 & 1,3930 \\
\hline $\mathrm{C}-\mathrm{Ca}$ & 0,7790 & 0,9371 & 1,7501 & 2,0930 & 1,4122 \\
\hline $\mathrm{C}-\mathrm{Mg}$ & 0,8337 & 1,0716 & 1,8655 & 2,0558 & 1,5590 \\
\hline $\mathrm{C}-\mathrm{S}$ & 0,9075 & 0,9435 & $1,2742 * *$ & $0,7577 * *$ & $0,2368 * *$ \\
\hline $\mathrm{C}-\mathrm{EM}$ & 0,8152 & 1,1127 & 1,7442 & 2,0788 & 1,5749 \\
\hline $\mathrm{N}$ & 0,7005 & 0,8283 & $1,1067 * *$ & $0,7253 * *$ & $0,2587 * *$ \\
\hline NP & 0,8161 & 0,9578 & $1,1277 * *$ & $0,7635 * *$ & $0,3258 * *$ \\
\hline NS & 0,7349 & 0,8316 & $1,4621 *$ & $2,2001 *$ & $1,5190 * *$ \\
\hline NPS & 0,7037 & 0,8871 & 1,9210 & 2,2219 & 1,5559 \\
\hline
\end{tabular}

B. Profunidade $20-40 \mathrm{~cm}$

\begin{tabular}{|c|c|c|c|c|}
\hline $\mathrm{T}$ & $0,2747 * *$ & $0,1863 * *$ & $0,2228 * *$ & $0,2023 * *$ \\
\hline $\mathrm{C}$ & 0,9428 & 1,6022 & 1,8989 & 1,2209 \\
\hline$C-P$ & $0,7097^{*}$ & $0,7681 * *$ & $1,1175^{* *}$ & $1,0071 * *$ \\
\hline$C-K$ & 0,9176 & 1,4426 & 1,8556 & 1,3678 \\
\hline $\mathrm{C}-\mathrm{Ca}$ & 0,9627 & 1,5262 & 1,9606 & 1,2751 \\
\hline $\mathrm{C}-\mathrm{Mg}$ & 1,0274 & 1,4506 & 1,9380 & 1,2318 \\
\hline$C-S$ & 0,8769 & $0,9918 * *$ & $0,6949 * *$ & $0,3497 * *$ \\
\hline$C-E M$ & 0,9342 & 1,4112 & 1,9365 & 1,2853 \\
\hline $\mathrm{N}$ & $0,7332 * *$ & $0,5884 * *$ & $0,3758 * *$ & $0,2754 * *$ \\
\hline NP & $0,8181 *$ & $0,7226 * *$ & $0,4860 * *$ & $0,2936 * *$ \\
\hline NS & $0,7944 * *$ & $0,8744 * *$ & $1,2488 * *$ & $1,0031 * *$ \\
\hline NPS & 0,9043 & $0,1863 * *$ & $0,2228 * *$ & $0,2023 * *$ \\
\hline
\end{tabular}

C-EM por lo que se infiere que los suelos estan bien provistos de estos elementos.

Se contempla que el tratamiento $\mathrm{T}$ posee diferencias significativas desde el primer corte, en ambos suelos y por su bajo rendimiento a partir del cuarto no se lo continuó evaluando. No aconteció lo mismo con los tratamientos C-P y $\mathrm{C}-\mathrm{S}$, en los cuales las deficiencias se advirtieron desde el segundo corte en ambas concentraciones y profundidades.

Por otro lado, los tratamientos aditivos confirman la existencia de carencias de los elementos $\mathrm{S}$ y $\mathrm{P}$, en este orden; siendo más relevantes las mismas en el suelo subsuperficial.

$\mathrm{Si}$ bien las deficiencias detectadas en $\mathrm{S}$ fueron importantes, incluso en trabajos preliminares (Galarza et al., 1993), la extracción intensa que realiza el Lolium de este nutriente en condiciones favorables para su desarrollo y en alta densidad de plantas por macetas, sobreestimaría los resultados con respecto a las condiciones de campo. No obstante ello, el secado de las muestras de suelo, favoreciendo la mineralización de los compuestos orgánicos más lábiles (Echeverría \& Navarro, 1983), tendería a equilibrar esta situación al menos hasta los primeros cortes y podría emplearse como argumento a favor de que es factible la deficiencia de este elemento en el campo.

Esta carencia se explicaría considerando que la fuente principal de $\mathrm{S}$ es la materia orgánica a través de la mineralización (Mengel \& Kirby, 1987; Lindemann et al., 1991) y que en condiciones aérobicas es fácilmente removido del perfil por lavado en forma de sulfatos (Mengel \& Kirby, 1987; Albrecht et al., 2000), favorecido por la textura (Echeverría \& Navarro, 1983) de estos suelos. También contribuye a esto la dinámica 
compleja que posee el suelo (Tomé Jr, 1997; Cordone et al., 1999).

Deficiencias de azufre han sido reportadas en varias partes del mundo incluyendo Africa, Australia, Nueva Zelandia y EEUU (Mengel \& Kirby, 1987). En Argentina varios autores reportaron deficiencias (Fisher et al. 1981; Echeverría \& Navarro, 1985; Pilatti et al., 1988; San Martin \& Echeverría, 1993; Ambrogio, 2001). Sin embargo muchos estudios conducidos para probar la efectividad de las fuentes de $\mathrm{P}$ y S para las pasturas (Lefroy et al., 1994; Ventimiglia, 2000) en condiciones de invernáculo y pastoreo (Bowdler \& Pigot, 1990; Blair et al., 1994; Dana et al., 1994), muestran que los compuestos que poseen sulfatos, contribuyen más rapidamente en el desarrollo de los cultivos que la forma de S elemental.

Ensayos de campo de los cultivos más importantes de la zona para el estudio de las respuestas a las combinaciones de los elementos N, P y S surgen como interrogantes que deberán ser respondidos en los próximos estudios.

Otra manera de analizar los resultados de este ensayo en macetas, es utilizando los diagramas de fertilidad, propuestos por Schenkel (1971) y utilizados por Echeverría \& Navarro (1983) y Barbosa et al. (1997). Estos denominan índice de rendimiento (IR) de un determinado tratamiento al cociente entre la producción acumulada de materia seca del tratamiento en cuestión y la del tratamiento completo (C), expresada en porcentaje. Como el crecimiento de las plantas responde a una función logarítmica, el gráfico consta de un sistema de coordenadas semilogarítmicas, en donde el IR se presenta en la ordenada y los cortes de MS del tratamiento C en la abcisa, con lo cual se expresa el IR de la fertilización incompleta como la fertilidad actual y el tratamiento $\mathrm{C}$ como la fertilidad potencial del suelo en consideración. Asimismo, señalan que se pueden establecer con precisión la intensidad y jerarquía de las carencias y que la pendiente de la recta del IR representa la velocidad de agotamiento del nutriente. La importancia de las deficiencias está evidentemente relacionada con el descenso de los rendimientos en MS provocados por los cortes sucesivos. Según estos autores, a través de numerosas experimentaciones, una disminución de la producción entre el 40 y $70 \%$ es una deficiencia neta; mientras que por encima de este último valor, la misma no es segura en el suelo.

En las Figuras 1A e B se presentan los diagramas de fertilidad de los tratamientos que manifestaron diferencias estadísticas significativas. A los efectos de comparación se coloca el T.

Los altos valores de IR obtenidos para los tratamientos C-K, C-Ca, C-Mg y C-EM confirman que estos elementos del suelo se encuentran en cantidades que no limitan la producción de MS del ray grass, incluso luego de varios cortes.

Se observó un comportamiento distinto con el S, en donde a partir del segundo corte, la declinación de la recta del diagrama es notoria, produciéndose carencias a partir del cuarto corte en el suelo superficial. En el suelo de mayor profundidad la disminución del rendimiento es más evidente, presentándose a partir del tercer corte. Este comportamiento del $\mathrm{S}$ ya es citado por otros autores (Echeverría \& Navarro 1983) y se debe al rápido consumo de las formas disponibles y al agotamiento de
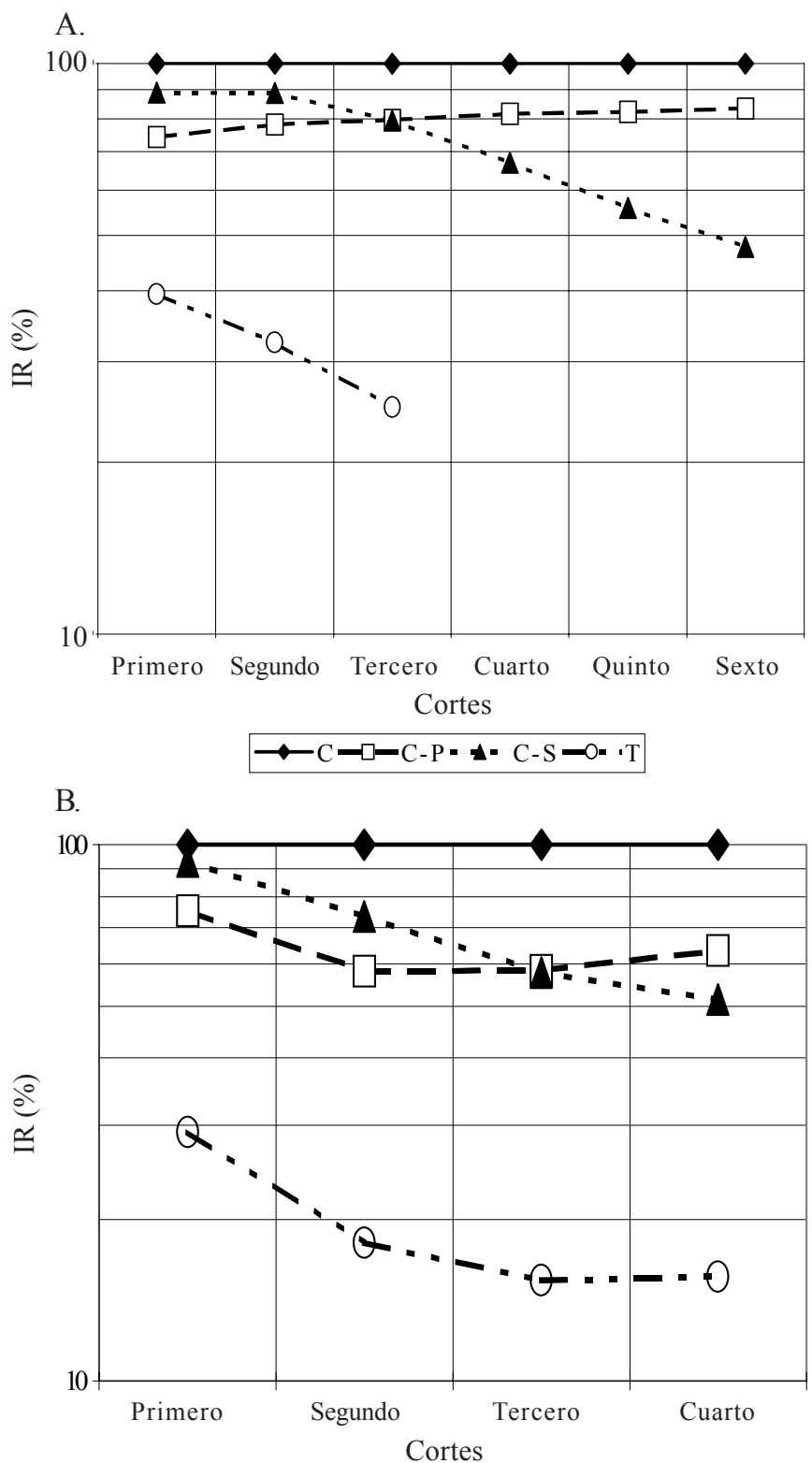

Figura 1. Indice de rendimiento (IR) los doferentes tratamientos del suelo $0-20 \mathrm{~cm}$ con concentración al $50 \%$ (A) y en suelo 20-40 cm con concentración al 33\% (B)

las fracciones orgánicas más lábiles susceptibles de ser mineralizadas.

Una situación intermedia se advierte en las curvas C-P que se estabilizan a partir del segundo y tercer corte, cuyos IR no fueron lo suficientemente bajos como para que exista una deficiencia del citado elemento en el suelo superficial. No sucede lo mismo en el suelo subsuperficial en donde dicha estabilización se logra por debajo del $70 \%$. Se puede pensar que esta estabilización de las curvas son debidas a la capacidad "buffer" de fosfatos que poseen estos suelos.

Con ambos análisis para la interpretación de los resultados se arriban a conclusiones similares, pero los diagramas de IR permitieron evaluar el grado de las deficiencias.

Se puede considerar que esta simple metodología en macetas utilizando ray grass bajo condiciones de invernáculo es útil para estimar deficiencias y prioridades de las mismas en el suelo. 


\section{CONCLUSIONES}

Los resultados muestram que cualquiera de las dos dosis utilizadas puede emplearse para la realización del trabajo, como ha sido expuesto; destacando la ventaja de la utilización de la concen-tración más baja para prevenir posibles desequilibrios iónicos en la solución del suelo.

A través de los datos obtenidos en los suelos estudiados se concluye que:

1. No existen deficiencias nutritivas de $\mathrm{K}, \mathrm{Mg}$, Ca ni EM, en las profundidades estudiadas.

2. Existe una deficiencia de $\mathrm{S}$, que se hace más evidente a partir del tercer corte.

3. El comportamiento del P no presenta una deficiencia segura, a pesar de que sus rendimientos presentaron diferencias altamente significativas.

\section{LITERATURA CITADA}

Albrecht R.E.; Vivas H.S.; Fontanetto H.; Hotian J.L. Residualidad del fósforo y del azufre en Soja sobre dos secuencias de cultivos. Campaña 1999/2000. En: Información Técnica de Soja y Maíz de segunda. INTA EEA Rafaela. 2000. p.? Publicación miscelánea 93

Barbosa O.A.; Galarza F.M.; Medina L.A.; Taboada M.A. Método simple de estimación de deficiencias nutritivas bajo condiciones de invernáculo. En: Congresso Brasileiro de Engenharia Agrícola, 26, 1997, Campina Grande. Resumos...Campina Grande: SBEA, CD Rom.

Blair G.J.; Dana M.; Lefroy R.D.B. A glasshouse evaluation of sulfur fertilizer sources for crops and pastures. A comparison of sulfur coated triple superphosphates and gypsum. Australian Journal of Agricultural Research, v.45, p.1517-1523, 1994.

Bowdler T.M.; Pigott F.J. The response of white clover to sulphur on an irrigated pasture in south-east Queensland. Tropical Grasslands, v.24, p.111-112, 1990.

Chaminade R. Diagnóstic des carences minerales du sol par l'experimentation en petites vases de vegetation. Science du Sol, p.157-168, 1964.

Cordone G.; Martinez F.; Abrate R. Fertilización azufrada. Agromercado, Cuadernillo Trigo No 34, Buenos Aires, 1999. p.2-6.

Dana M.; Lefroy R.D.B.; Blair G.J. A glasshouse evaluation of sulfur fertilizer sources for crops and pastures. Flooded and non-flooded rice. Australian Journal of Agricultural Research, v. 45, p. 1497-1515, 1994.

d'Hiriart A.; Marchi A. Pasto llorón Cv Tanganika. Efecto de la fertilización nitrogenada sobre la producción y calidad. Revista Argentina de Producción Animal, v.6, p.336-343, 1978.

Echeverría H.E. Exploración de deficiencias nutritivas en suelos agrícolas del sudeste bonaerense. II Fertilización N-P-S. Revista de Investigaciones Agropecuarias INTA, v.20, n.2, p.25-35, 1985.
Echeverría H.E.; Navarro C.A. Exploración de deficiencias nutritivas en suelos agrícolas del sudeste bonaerense. Método de Chaminade. Revista de Investigaciones Agropecuarias INTA, v.18, n.1, p.17-29, 1983.

Fisher H.G.; Mendoza R.; von Bertoldi A. Ajuste de una técnica sustrativa para el diagnóstico de posibles insuficiencias de nutrimentos minerales de suelos de la región central de Córdoba. Revista de Ciencias Agropecuarias (U.N.C.), v.11, p.41-53, 1981 .

Galarza F.M., Barbosa O.A., Peña Zubiate C.A.; Medina L.A.; Ruiz O.M.; Cerdá R.A. Deficiencias nutritivas en suelos del área de influencia de Villa Mercedes (SL). En: Congreso Argentino de Ciencia del Suelo,14, 1993, Mendoza. Actas... Mendoza: Asociación Argentina de la Ciencia del Suelo, 1993. p.175-176.

Jones J.B.; Case V.W. Sampling, handling and analyzing plant tissue. En: Westerman, R.L. (ed.) Soil testing and plant analysis. 3. ed. Madison: Soil Science Society of America. 1990. p.389-428.

Lefroy R.D.B.; Dana M.; Blair G.J. A glasshouse evaluation of sulfur fertilizer sources for crops and pastures. Soluble and non-soluble sulfur and $\mathrm{P}$ sources for pastures. Australian Journal of Agricultural Research, v.45, p.1525-1537, 1994.

Lindemann W.C.; Aburto J.J.; Haffner W.M.; Bono A.A. Effect of sulfur source on sulfur oxidation. Soil Science Society of American Journal, Madison, v.55, p.85-90, 1991.

Mengel K.; Kirby E.A. Principles of plant nutrition. Bern: International Potash Institute, 1987. 687p.

Pilatti M.A.; de Orellana J.A; Priano L.J.; Felli O.M.; Grenon D.A. Incidencia de manejos tradicionales y conservacionistas sobre propiedades físicas, químicas y biológicas de un Argiudol en el Sur de Santa Fé. Revista de la Asociación Argentina de la Ciencia del Suelo, v.6, p.19-29, 1988.

Priano L.J.; Pilatti M.A.; de Orellana J.A. Ensayo sobre deficiencia de nutrimentos en Argiudoles del Centro y Noreste de la provincia de Santa Fé. Comunicaciones Técnicas MG-FAVE, 1983, n.1, 10p.

Romero T; Bariggi C.; Schenkel G. Resultados preliminares de exploración de deficiencias nutritivas para suelos pampeanos en macetas con alfalfa. Proyecto Alfalfa-FAO-INTA Arg. 71/548, 1973. 12p.

San Martín N.F.; Echeverría H.E. Relevamiento de los sulfatos en suelos del sudeste bonaerense. En: Congreso Argentino de la Ciencia del Suelo, 14, 1993, Mendoza. Actas... Mendoza: Asociación Argentina de la Ciencia del Suelo, 1993, p.215-216.

Schenkel G. Evaluación de la fertilidad de un suelo, mediante la producción de materia seca en ensayos de macetas. II Diagrama de fertilidad. Turrialba, San Juan, v.21, p.263-271, 1971.

Sims J.T. Soil fertility evaluation. In: Summer, M.E. (ed). Handbook of soil science. Boca Raton: CRC Press. 2000.

Tomé Jr. J.B. Manual para interpretaçâo de análise de solo. 1. ed.Guaíba: Libraría e Editora Agropecuaria Ltda.,1997. 247p.

Ventimiglia L.; Carta H.; Rillo S. Azufre: la rotación también lo aprovecha. Cosecha gruesa, Campaña 1999/2000: Resultados de experiencias. UEEA INTA 9 de julio. Buenos Aires, Argentina. 2000. 